\title{
Programas de promoção da atividade física no Sistema Único de Şaúde brasileiro: revisão sistemática
}

\author{
Primary health care programs for physical activity promotion in the Brazil: \\ a systematic review
}

\author{
Leonardo Augusto Becker 1,2, Priscila Bezerra Gonçalves 1,2, Rodrigo Siqueira Reis 1,2,3
}

\section{Resumo}

O objetivo desta revisão foi sintetizar as evidências disponíveis na literatura referentes aos programas de promoção de atividade física (AF) no Sistema Único de Saúde brasileiro. Foi realizada uma busca sistemática da literatura nas bases de dados PubMed, Science Direct, Biblioteca Virtual de Saúde (BVS), base de dados de dissertações e teses e uma busca manual na Revista Brasileira de Atividade Física e Saúde (RBAFS) no período entre janeiro de 2005 à abril de 2015. Foram incluídos estudos: originais, realizados no Brasil, com desfecho a promoção da AF realizada no Sistema Único de Saúde em idiomas: Inglês, Espanhol e/ou Português. A análise final foi composta por 17 artigos. Desses, $29,41 \%(n=5)$ foram realizados em todo o território nacional e 5,88\% (n=1) em duas ou mais regiões do país. Observou-se ainda uma disparidade regional, com 29,41\% $(n=5)$ dos estudos na região sudeste, $17,64 \%(n=3)$ na região sul, $11,76 \%(\mathrm{n}=2)$ na região nordeste, apenas 5,88\% $(\mathrm{n}=1)$ na região centro-oeste e nenhum estudo foi realizado na região norte do país. Enquanto ao modo de intervenção verificouse que $47,05 \%(n=8)$ ocorreram no Sistema Único de Saúde e $47,05 \%(n=8)$ foram programas com equipe multiprofissional. Os estudos, em sua maioria forneceram dados descritivos ou sobre resultados na AF ou qualidade de vida, sendo escassas as informações sobre processo e impacto dos programas. Os programas com equipe multiprofissional foram estratégias importantes de promoção de AF. Sugere-se realizar estudos com delineamentos longitudinais ou mesmo experimentais, acerca do custo e benefício dos programas implementados.

\section{Palavras-chave}

Exercício; Atividade motora; Atenção primária de saúde; Promoção da saúde; Brasil.

\begin{abstract}
The objective of this review was to synthesize the available evidence in the literature related to Physical Activity promotion programs (PA) primary health care System in the Brazilian. A systematic literature search in PubMed, Science Direct, Virtual Health Library, database of dissertations and theses and manual search in the Journal of Physical Activity and Health between January 2005 to April 2015. For selection the studies were: original, made in Brazil, with the outcome to promote PA held in the national health system languages: English, Spanish and / or Portuguese. The final analysis consisted of 17 articles. Of these, $29.41 \%(n=5)$ were held across the country and $5.88 \%(n=1)$ in two or more regions. It was also observed regional disparity, with $29.41 \%(n=5)$ of studies the Southeast, $17.64 \%(n=3)$ the South, $11.76 \%(n=2)$ in the northeast, only $5.88 \%(n=1)$ the Midwest and no study was conducted in the northern region of the country. While the intervention it was found that $47.05 \%(n=8)$ occurring in the Unified Health System and $47.05 \%(n=8)$ were programs with multidisciplinary team. Studies, mostly provided descriptive data or results on the PA or quality of life, and little information about the process and impact of the programs. The programs with the multidisciplinary team were important strategies PA promotion. It is suggested to carry out studies with longitudinal or even experimental designs, about the costs and benefits of the implemented programs.
\end{abstract}

\section{Keywords}

Exercise; Motor Activity; Primary Health Care; Health Promotion; Brazil.
1 Pontifícia Universidade Católica do Paraná. Grupo de Pesquisa em Atividade Física e Qualidade de Vida (GPAQ). Curitiba-PR, Brasil.

2 Universidade Federal do Paraná. Programa de Pós-Graduação em Educação Física, Curitiba-PR, Brasil

3 Pontifícia Universidade Católica do Paraná. Programa de Pós Graduação em Gestão Urbana (PPGTU). Curitiba-PR, Brasil.

\section{Introdução}

A inatividade física caracteriza-se como uma "pandemia” que atinge um em cada três adultos em todo o globo ${ }^{1}$, sendo responsável por 5,3 milhões de mortes no mundo ${ }^{2}$. Tal magnitude torna a inatividade física 
a quarta principal causa de mortes em todo o mundo ${ }^{3}$. Neste contexto, programas que promovam a prática da atividade física $(\mathrm{AF})$ tem recebido grande atenção, principalmente no sistema público de saúde, pois entre $1 \%$ e $2,6 \%$ de todos os gastos em saúde, em países de alta renda, estão relacionados com a inatividade física ${ }^{4}$. De fato, intervenções no sistema de saúde apresentam efeitos positivos nos níveis de $\mathrm{AF}^{5,6}$ principalmente ações educativas (guias, diretrizes) ${ }^{7,8}$, aconselhamento (individual ou em grupo) 7,8 e mudanças de comportamento (hábitos alimentares e comportamento sedentário) $)^{5,8}$.

No Brasil, a AF tem feito parte das agendas de promoção de saúde através da "Política Nacional de Promoção de Saúde (PNPS)", e de prevenção de Doenças Crônicas Não Transmissíveis (DCNTs), nas quais, diversas ações e metas têm sido estabelecidas ${ }^{9,10}$. Tais ações, em grande medida, são inseridas dentro do Sistema Único de Saúde (SUS) e integram o conjunto de iniciativas da Atenção Primária em Saúde (APS) ${ }^{9,10}$ que, por meio desta, participa de programas de controle do álcool, tabaco, alimentação saudável e $\mathrm{AF}^{11}$.

A promoção da AF na APS tem sido uma das ações prioritárias na comunidade, em especial para as populações de baixa e média rendas ${ }^{11,12}$. Porém, a rápida expansão e aceitação destes programas ${ }^{13,14}$ não tem sido acompanhada da avaliação e compreensão sobre as características, os tipos de ações, implementação, resultados e outros aspectos que compõem estas iniciativas. Neste sentido, é importante mapear as publicações sobre esta temática e obter informações sobre tais programas, pois tal conhecimento poderá auxiliar gestores e profissionais a aprimorar as práticas para implementação dos programas de promoção de AF na APS. Sendo assim, este estudo tem como objetivo sintetizar as evidências disponíveis na literatura revisada por pares referentes aos programas de promoção de AF realizados no Sistema Único de Saúde brasileiro.

\section{Métodos}

O presente estudo utilizou os critérios metodológicos estabelecidos pelo guia Preferred Reporting Items for Systematic Reviews and Meta-Analyses (PRISMA) ${ }^{15}$. Foi realizada uma ampla revisão da literatura para identificar os potenciais descritores que pudessem auxiliar na seleção dos artigos e responder aos objetivos deste estudo. Para estratégia de busca sistemática, optou-se por utilizar os Descritores em Ciências da Saúde (DeCS). Desta maneira, os termos encontrados foram testados individualmente e por fim chegou-se a combinação: "exercise" OR "motor activity" AND "primary health care" OR "delivery health care" OR "bealth promotion" AND "Brazil". Todos os descritores foram utilizados em inglês.

A busca foi realizada em três (03) bases de dados, BIBLIOTECA VIRTUAL DE SAÚDE (BVS) (que inclui LILACS, MEDLINE,MEDCARIB, OPAS/OMS, PAHO E WHOLIS), SCIENCE DIRECT, PUBMED. Em seguida foi realizada uma busca nas bases de dados de teses e dissertações. Posteriormente uma busca manual na Revista Brasileira de Atividade Física e Saúde (RBAFS), pois esta não estava indexada nas bases de dados selecionadas, mas é um periódico específico para a temática do estudo, possuindo elevado número de publicações na área de promoção da AF. Para todas as estratégias de busca optou-se por realizá-las no período de janeiro de 2005 até abril de 2015.

Para a inclusão dos artigos, foram estabelecidos os seguintes critérios: a) Estudos originais; b) Realizados no Brasil; c) Considerando como desfecho a promoção da AF no SUS, ou seja, todo o programa de AF que foi concebido, gerenciado ou executado no contexto do SUS. A partir deste conceito, os artigos foram ras- 
treados para verificar informações sobre concepção, coordenação e execução que estavam vinculados ao SUS, como por exemplo; execução Unidades Básicas de Saúde (UBS) e coordenação (Secretaria de saúde e Secretaria de esporte, lazer e juventude), d) Idiomas: Inglês, Espanhol e/ou Português; e) Dissertações e teses; f) Publicados a partir de 2005, uma vez que foi neste ano que teve inicio a liberação de recursos para promoção da AF no SUS ${ }^{16}$. Foram excluídos os estudos de revisão (narrativas, sistemáticas e/ou meta análises), relatórios e monografias. Dois pesquisadores realizaram de maneira independente cada etapa do processo de revisão. Caso houvesse divergência no processo de inclusão e exclusão, foi realizada uma reunião de consenso entre os pesquisadores.

Inicialmente todos os títulos selecionados nas bases de dados $(\mathrm{n}=1.627)$ foram transferidos para o software EndNote, em seguida foram excluídos os títulos repetidos ( $\mathrm{n}=69)$. O processo de revisão sistemática foi composto por cinco fases. A primeira fase consistia pela leitura dos títulos, dos 1.558 (100\%) títulos encontrados, 1.235 foram excluídos por não apresentarem relação com o tema, 48 por se tratarem de revisões ou artigos metodológicos e 36 foram realizados em outros países. $\mathrm{Na}$ segunda fase foi realizada a leitura dos resumos ( $n=239 ; 15,34 \%)$, destes 158 foram excluídos por não apresentarem relação com o tema, 20 artigos de revisão ou metodológico, 15 que não apresentaram como desfecho a promoção de AF no SUS, 11 editoriais e três por outros motivos (dois relatórios e um realizado em outro país). $\mathrm{Na}$ terceira fase foi realizada a leitura na íntegra dos artigos restantes juntamente com sete artigos que foram inseridos manualmente através da busca na RBAFS $(\mathrm{n}=39 ; 2,50 \%)$. Destes, 22 artigos foram excluídos, sendo 11 por não apresentaram como desfecho a promoção de AF no SUS, seis realizados em outros países, quatro de revisão ou metodológico e um artigo que não estava disponível para leitura. Ao final, 17 (1,09\%) artigos atenderam aos critérios empregados e foram selecionados para revisão, análise e descrição metodológica. A Figura 1 apresenta o processo de busca aos artigos, os resultados e os respectivos motivos de exclusão dos mesmos.

Após a seleção e leitura completa dos artigos, os resultados foram descritos contendo as informações gerais dos estudos (autor, ano, delineamento, localidade, amostra, faixa etária, tipo de intervenção, principais resultados e o modo de avaliação) (Tabela 1).

Em seguida, foram classificados os tipos de intervenção. Para tanto, foram utilizados os critérios adotados pela International Society for Physical Activity and Health $^{17}$, descrevendo os sete melhores investimentos que funcionam para promoção da AF, sendo: 1) programas no contexto escolar; 2) políticas e sistemas que priorizem caminhadas, uso de bicicletas e transporte público; 3) Desenho urbano e criação de locais seguros para prática da AF; 4) Promoção da AF e prevenção de doenças crônicas não transmissíveis junto ao SUS; 5) Campanhas educativas na mídia que promovam a prática da AF; 6) Desenvolvimento de programas com equipe multiprofissional; 7) Programas que busquem através do esporte, contribuir e encorajar a prática de AF na comunidade.

Em relação ao tipo de avaliação dos programas, os artigos foram classificados em quatro categorias: a) artigos que apresentaram apenas informações descritivas; b) processo de implementação do programa (avaliação dos profissionais, materiais de divulgação, infraestrutura, metodologia e recursos do programa); c) impacto do programa (conhecimento do programa, mudanças de comportamento dos frequentadores e delineamento dos estudos); d) resultados do programa (percepção de qualidade de vida e aumento nos níveis de AF dos frequentadores), a partir da informações disponíveis nos artigos ${ }^{18}$. 


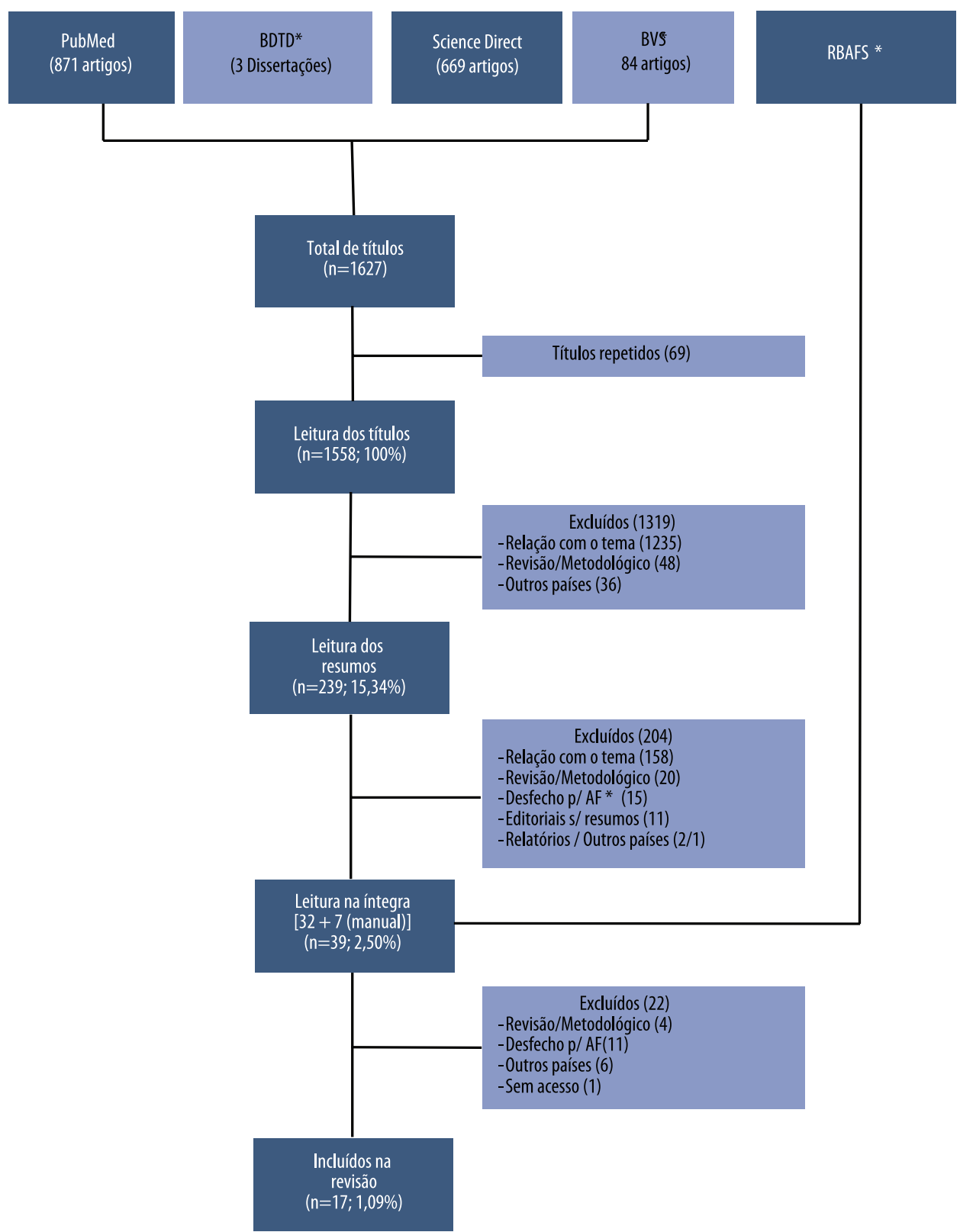

FIGURA 1 - Fluxograma de identificação e seleção dos artigos para revisão sistemática sobre promoção de atividade física.

*(BVS) Biblioteca Virtual de Saúde, (RBAFS) (Revista Brasileira de Atividade Física e Saúde, (BDTD) Bases de Dados de Dissertações e Teses, (AF) Atividade Física.

TABELA 1 - Estudos no Sistema Único de Saúde para promoção da atividade física no Brasil segundo o autor, ano, delineamento, localidade, amostra, faixa etária, tipo de intervenção, modo de avaliação e os principais resultados. Período de 2005-2015.

\begin{tabular}{|c|c|c|c|c|c|c|c|}
\hline Autor, Ano & ${ }^{*}$ Del & $\begin{array}{l}\text { Localidade } \\
\text { (Cidade/Estado) }\end{array}$ & Amostra & $\begin{array}{l}\text { Faixa etária } \\
\text { (anos) }\end{array}$ & $\begin{array}{l}\text { Tipo de } \\
\text { intervenção }\end{array}$ & $\begin{array}{l}\text { Modo de } \\
\text { Avaliação }\end{array}$ & Principais resultados \\
\hline Nakamura ${ }^{27}$ & L & Rio Claro, SP & $\begin{array}{l}440 \text { ( } 409 \text { mulheres e } \\
31 \text { homens) }\end{array}$ & $\geq 20$ anos & 4 & Resultados & $\begin{array}{l}\text { Ao término do programa participaram } 440 \\
\text { participantes ( } 409 \text { mulheres com média } \\
\text { de idade } 50 \pm 26 \text { anos e } 31 \text { homens média } \\
\text { de idade } 64 \pm 10 \text { anos). Todas as variáveis } \\
\text { de aptidão física (coordenação motora, } \\
\text { agilidade e força muscular) apresentaram } \\
\text { melhoras significativas para os participantes } \\
\text { com idade } \geq 70 \text { anos exceto a capacidade } \\
\text { aeróbica e flexibilidade. A capacidade aeróbia, } \\
\text { flexibilidade e agilidade teve diferença } \\
\text { significativa quanto ao sexo (homens) ( } p \leq 0,05 \text { ) }\end{array}$ \\
\hline
\end{tabular}




\begin{tabular}{|c|c|c|c|c|c|c|c|}
\hline Autor, Ano & *Del & $\begin{array}{l}\text { Localidade } \\
\text { (Cidade/Estado) }\end{array}$ & Amostra & $\begin{array}{l}\text { Faixa etária } \\
\text { (anos) }\end{array}$ & $\begin{array}{l}\text { Tipo de } \\
\text { intervenção }\end{array}$ & $\begin{array}{l}\text { Modo de } \\
\text { Avaliação }\end{array}$ & Principais resultados \\
\hline Gomes $^{19}$ & $\mathrm{~T}$ & Brasil $^{* *}$ & $\begin{array}{l}1.251 \text { coordenadores } \\
\text { de unidades de saúde }\end{array}$ & $\geq 18$ anos & 4 & Descritivo & $\begin{array}{l}\text { Entre as unidades que possuíam programas } \\
\text { de intervenção para AF a maior proporção } \\
\text { foi encontrada na região sudeste }(50,3 \%) \text {, } \\
\text { diferindo das outras regiões (sul, norte, } \\
\text { nordeste e centro-oeste) }(p<0,01) \text {. }\end{array}$ \\
\hline Lopes $^{31}$ & T & $\begin{array}{l}\text { Belo Horizonte, } \\
\text { MG }\end{array}$ & 1.616 & $\geq 20$ anos & 4 & Resultados & $\begin{array}{l}\text { Os médicos ( } 69,0 \%) \text { foram os principais } \\
\text { responsáveis pelo aconselhamento, seguidos } \\
\text { por enfermeiros }(6,1 \%) \text {. Ter recebido } \\
\text { aconselhamento foi mais frequente entre } \\
\text { os indivíduos com } 50 \text { a } 59 \text { anos; do sexo } \\
\text { feminino; com maior renda e escolaridade; que } \\
\text { apresentaram ao menos uma DCNT( } p \leq 0,05) \text {. }\end{array}$ \\
\hline Papini ${ }^{28}$ & GE & Rio Claro, SP & 13 Mulheres & $\geq 35$ anos & 4 & Resultados & $\begin{array}{l}\text { Após um ano, não foi verificado mudanças } \\
\text { significativas no peso, índice de massa corporal } \\
\text { ou circunferência da cintura. }\end{array}$ \\
\hline Ramos 11 & $\mathrm{~T}$ & Brasil** & $\begin{array}{l}1.600 \text { coordenadores, } \\
\text { médicos e } \\
\text { enfermeiros de } \\
\text { unidades de saúde }\end{array}$ & $\geq 18$ anos & 4 & Descritivo & $\begin{array}{l}\text { A promoção de atividade física esteve presente } \\
\text { em } 39,8 \% \text { das unidades básicas de saúde. As } \\
\text { atividades mais realizadas foram caminhadas } \\
\text { em grupo, exercícios de força e atividades } \\
\text { de relaxamento. Os programas de atividades } \\
\text { físicas estavam mais frequentes na região } \\
\text { sudeste do país } 50,9 \% \text { e menor na região } \\
\text { norte }(p<0,01) \text {. }\end{array}$ \\
\hline Reis ${ }^{\mathbf{2 3}}$ & $\mathrm{T}$ & Vitória, ES & 2.023 & $\geq 18$ anos & 6 & $\begin{array}{l}\text { Impacto e } \\
\text { Resultados }\end{array}$ & $\begin{array}{l}31,5 \% \text { dos entrevistados relataram conhecer } \\
\text { o programa e apenas } 1,5 \% \text { dos participavam } \\
\text { do programa, sendo a maior proporção de } \\
\text { mulheres ( } p<0,05) \text {. }\end{array}$ \\
\hline Amorin ${ }^{14}$ & $\mathrm{~T}$ & Brasil** & $\begin{array}{l}748 \\
\text { coordenadores } \\
\text { (RNAF) }\end{array}$ & $\geq 18$ anos & 6 & Descritivo & $\begin{array}{l}\text { A região centro-oeste possuía, em maior } \\
\text { número, cidades com programas de promoção } \\
\text { da } A F \text {, seguida pela região sudeste do país. }\end{array}$ \\
\hline Florindo ${ }^{12}$ & $\mathrm{~T}$ & Brasil** & $\begin{array}{l}529 \text { (182 médicos e } \\
347 \text { enfermeiras) }\end{array}$ & $\geq 18$ anos & 4 & Descritivo & $\begin{array}{l}\text { A prevalência de aconselhamento para a } \\
\text { prática de atividade física nos últimos seis } \\
\text { meses foi de } 68,9 \% \text {, sendo mais frequente } \\
\text { entre os médicos }(81,2 \%) \text { seguida pelos } \\
\text { enfermeiros }(62,4 \%) \text {. }\end{array}$ \\
\hline Giraldo 29 & $\mathrm{~T}$ & Rio Claro, SP & 26 Mulheres & $\begin{array}{l}\geq 18 \text { anos } \\
\text { (média de } \\
\text { idade } 53,9 \\
\text { anos) }\end{array}$ & 4 & Resultados & $\begin{array}{l}\text { A maioria das mulheres residiam próximos aos } \\
\text { locais de intervenção. A média de participação } \\
\text { foi } 23 \text { meses ( } D P=6) \text { e a média de AF foi de } \\
98 \text { minutos semanais ( } D P=108,4) \text {. O principal } \\
\text { grupo de risco atendido foram as hipertensas } \\
(53,8 \%) \text {. Após a intervenção as participantes } \\
\text { relataram que a percepção de saúde esteve } \\
\text { melhorada, houve a redução de pressão arterial } \\
\text { e redução da glicemia. }\end{array}$ \\
\hline Ferreira ${ }^{30}$ & $\mathrm{~T}$ & Goiânia, GO & 911 Adolescentes & $\begin{array}{l}13 \text { a } 18 \\
\text { anos }\end{array}$ & 1 & Resultados & $\begin{array}{l}\text { Foram entrevistados } 911 \text { adolescentes, sendo } \\
492(54 \%) \text { do sexo masculino. Tanto no grupo } \\
\text { controle como intervenção a maioria dos } \\
\text { adolescentes eram inativos ou fisicamente } \\
\text { ativos ( } p>0,05) \text {. Em relação ao consumo de } \\
\text { alimentos protetores, não houve associação } \\
\text { entre o grupo controle e intervenção }(p>0,05)\end{array}$ \\
\hline Hallal 21 & $\mathrm{~T}$ & Recife, PB & 554 (50\% usuários) & $\geq 16$ anos & 6 & $\begin{array}{l}\text { Impacto e } \\
\text { Processo }\end{array}$ & $\begin{array}{l}\text { A maioria dos usuários, ficaram sabendo } \\
\text { do programa por ter visto um polo (65\%) } \\
\text { ou por meio de outra pessoa ( } 28,9 \%) \text {. Os } \\
\text { frequentadores ( } 72,8 \% \text { ) estavam satisfeitos } \\
\text { com o programa, porém } 61 \% \text { relataram que } \\
\text { gostariam de uma melhor estrutura, aquisição } \\
\text { de novos matérias, } 2,3 \% \text { sugeriram contratação } \\
\text { de profissionais e maior divulgação, } 8,7 \% \\
\text { gostariam da ampliação do serviço. Entre os } \\
\text { usuários, a principal razão para participarem no } \\
\text { programa foi melhorar a sua saúde }(65,7 \%) \text {. } 0 \\
\text { tempo médio de participação foi de } 27,5 \text { meses } \\
\text { (DP=23,1), a média diária foi de } 69 \text { minutos } \\
\text { (DP=22,3) e } 72,8 \% \text { relataram alta satisfação. }\end{array}$ \\
\hline
\end{tabular}




\begin{tabular}{|c|c|c|c|c|c|c|c|}
\hline Autor, Ano & *Del & $\begin{array}{l}\text { Localidade } \\
\text { (Cidade/Estado) }\end{array}$ & Amostra & $\begin{array}{l}\text { Faixa etária } \\
\text { (anos) }\end{array}$ & $\begin{array}{l}\text { Tipo de } \\
\text { intervenção }\end{array}$ & $\begin{array}{l}\text { Modo de } \\
\text { Avaliação }\end{array}$ & Principais resultados \\
\hline Reis ${ }^{25}$ & $\mathrm{~T}$ & Curitiba, PR & 2.097 & $\geq 16$ anos & 6 & $\begin{array}{l}\text { Impacto e } \\
\text { Resultados }\end{array}$ & $\begin{array}{l}\text { Cerca de } 91,6 \% \text { dos entrevistadores conheciam } \\
\text { o programa e 5,6\% participavam das atividades } \\
\text { oferecidas. A participação do programa foi } \\
\text { associada com o sexo (mulheres) e idade (+55 } \\
\text { anos) (p<0,01). Os participantes que relataram } \\
\text { participar ou conhecer o programa eram } \\
\text { fisicamente ativos no tempo livre e em caminhadas, } \\
\text { quando comparados com aqueles que não } \\
\text { participam ou não conheciam o programa. }\end{array}$ \\
\hline Knuth ${ }^{13}$ & $\mathrm{~T}$ & Brasil & $\begin{array}{l}225 \text { coordenadores } \\
\text { (RNAF) }\end{array}$ & $\geq 18$ anos & 6 & Descritivo & $\begin{array}{l}\text { A maioria dos projetos estava vinculado a ESF, } \\
\text { sendo que } 35,1 \% \text { desenvolvem atividades em } \\
\text { parceria com os NASF. }\end{array}$ \\
\hline Hallal ${ }^{22}$ & $\mathrm{~T}$ & Curitiba, PR & 120 & $\geq 18$ anos & 6 & $\begin{array}{l}\text { Processo e } \\
\text { Impacto }\end{array}$ & $\begin{array}{l}\text { Dos entrevistados, } 75,8 \% \text { relataram já ter visto } \\
\text { o programa, 15\% participaram, } 48 \% \text { realizavam } \\
\text { AF nos espaços ofertados pela PMC. Apenas } \\
10 \% \text { relataram ter recebido algum folder do } \\
\text { Programas CuritibAtiva. Mais da metade dos } \\
\text { entrevistaram atribuíram ótima ou boa a } \\
\text { avaliação dos itens dos programas. }\end{array}$ \\
\hline Siqueira ${ }^{20}$ & $\mathrm{~T}$ & $\begin{array}{l}\text { Região Sule } \\
\text { Região nordeste } \\
* * *\end{array}$ & $\begin{array}{l}8.063 \\
\text { Usuários do sistema } \\
\text { único de saúde }\end{array}$ & $\geq 30$ anos & 4 & Descritivo & $\begin{array}{l}\text { Cerca de }(28,9 \%) \text { dos adultos e }(38,9 \%) \text { dos } \\
\text { idosos receberam aconselhamento para } \\
\text { prática da atividade física nas unidades } \\
\text { básicas de saúde, sendo a maior prevalência } \\
\text { na região nordeste do país. Nas unidades } \\
\text { de saúde modelo ESF verificou-se uma } \\
\text { maior prevalência de aconselhamento de AF } \\
\text { independentemente da faixa etária quando } \\
\text { comparado com a UBS tradicional ( } p<0,001) \text {. }\end{array}$ \\
\hline Simões ${ }^{24}$ & $\mathrm{~T}$ & Recife, PB & $\begin{array}{l}2.038 \text { moradores da } \\
\text { cidade de Recife }\end{array}$ & $\geq 16$ anos & 6 & Resultados & $\begin{array}{l}\text { Os ex-participantes do programa academia } \\
\text { da cidade eram duas vezes mais propensos a } \\
\text { praticarem AFMV no lazer quando comparado com } \\
\text { aqueles que nunca participaram do programa. }\end{array}$ \\
\hline Gomes $^{26}$ & GE & Florianópolis, SC & $\begin{array}{l}103 \text { Grupo } \\
\text { Experimental (51) e } \\
\text { Grupo Controle (52) }\end{array}$ & $\begin{array}{l}\geq 18 \text { anos } \\
\text { (média de } \\
\text { idade } 47 \\
\text { anos) }\end{array}$ & 6 & Resultados & $\begin{array}{l}\text { Após intervenção o grupo experimental, } \\
\text { observou-se uma diferença significativa na } \\
\text { percepção de saúde e o nível de atividade física } \\
\text { (total) (p>0,05). No grupo controle não foram } \\
\text { observadas mudanças significativas na prática } \\
\text { de atividade física habitual. }\end{array}$ \\
\hline
\end{tabular}

*Delineamento: T(Transversal), GE(Grupo Experimental), L(Longitudinal) Local: RS(Rio Grande do Sul), SC(Santa Catarina), PR (Paraná), SP (São Paulo), MG (Minas Gerais), GO (Goiânia), PB (Pernambuco), SE (Sergipe), Brasil (todas as regiões do país). Tipo de intervenção: 1) programas no contexto escolar; 2) políticas e sistemas que priorizem caminhadas, uso de bicicletas e transporte público; 3) Desenho urbano e criação de locais seguros para prática da AF; 4) Promoção da AF e prevenção de doenças crônicas não transmissíveis junto ao sistema único de saúde; 5) Campanhas educativas na mídia que promovam a prática da AF; 6) Desenvolvimento de programas com equipe multiprofissional; 7) Programas que busquem através do esporte, contribuir e encorajar a prática de AF na comunidade. Principais resultados: DCNT (Doenças Crônicas Não Transmissíveis), AFL (Atividade Física no Lazer), PAC (Programa Academia da Cidade), ESF (Estratégia Saúde da Família), NASF (Núcleo de Apoio da Saúde da Família), UBS (Unidade Básica de Saúde), AF(Atividade Física), AFMV (Atividade Física Moderada e Vigorosa).

\section{Resultados}

Combinando-se todas as estratégias de buscas, foram identificados 1.627 estudos relevantes. Após a exclusão dos artigos duplicados entre as bases de dados, leitura dos títulos e resumos, foi realizada a leitura na íntegra de 39 estudos. No total, 17 estudos contemplaram os critérios de inclusão, sendo que destes cinco foram selecionados de maneira manual através da busca na RBAFS. O maior número de publicações ocorreu nos anos de $2014(n=5)$ e 2013 (n=4) (Figura 2).

No que se refere à faixa etária, a população adulta ( $\geq 18$ anos), foi a mais frequente $(76,47 \%)$ e apenas um artigo foi realizado com adolescentes em amostra de base escolar (Tabela 1 ). 


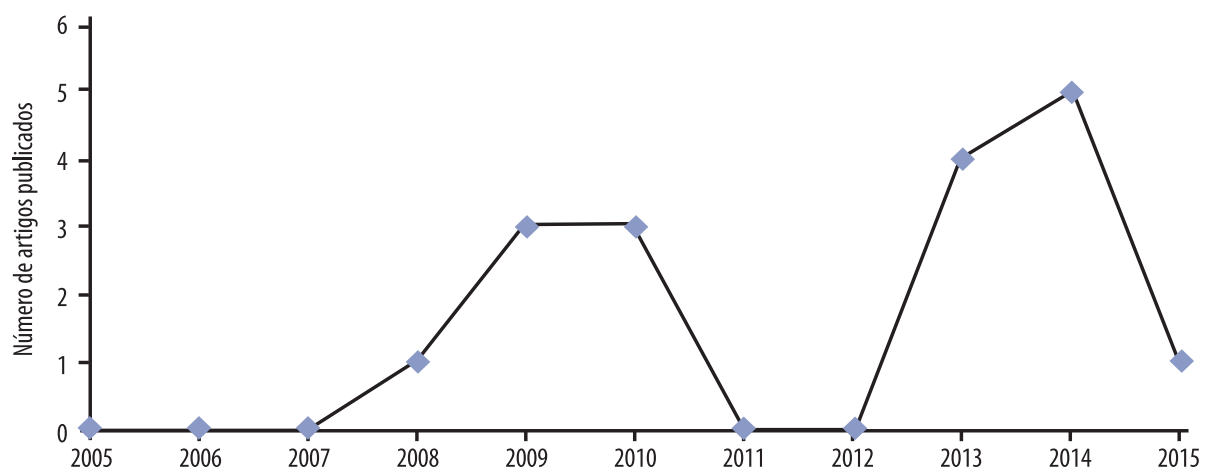

FIGURA 2 - Número de artigos publicados anualmente no período de 2005 a 2015.

Em relação a local de execução dos estudos aproximadamente 1/3 (29,41\%; $\mathrm{n}=5$ ) foram realizados em todo o território nacional e 5,88\% (n=1) em duas ou mais regiões do país. Observou-se ainda disparidade regional, com 29,41\% ( $\mathrm{n}=5$ ) dos estudos na região sudeste, $17,64 \%(\mathrm{n}=3)$ na região sul, $11,76 \%(\mathrm{n}=2)$ na região nordeste, apenas 5,88\% $(\mathrm{n}=1)$ na região centro-oeste e nenhum estudo foi realizado na região norte do país (Figura 3 ).

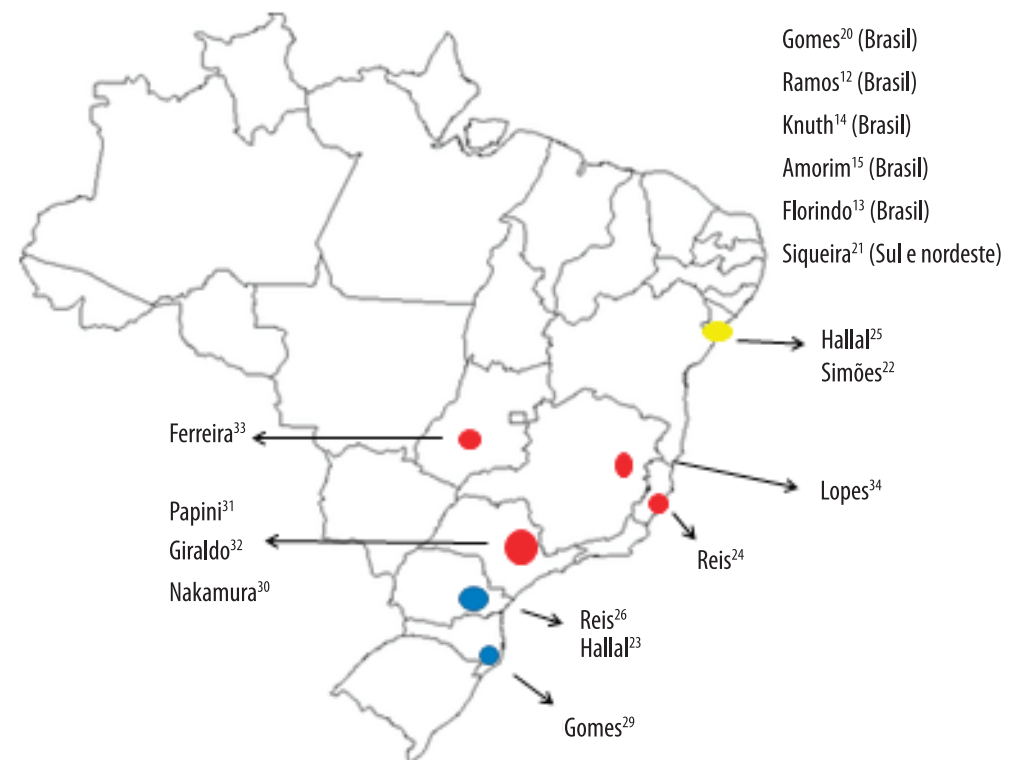

FIGURA 3 - Estudos incluídos na revisão sistemática de acordo com a região do País.

Enquanto ao tipo de intervenção, observou-se que 47,05\% (n=8) foram ações de promoção da AF e prevenção de DCNTs junto ao SUS, 47,05\% (n=8) eram programas com equipe multiprofissional e 5,88\% $(n=1)$ dos estudos relataram ações desenvolvidas na escola (Tabela 1). Em todos os estudos foram utilizados questionários como instrumento de avaliação da AF. Nos programas com equipe multiprofissional o principal questionário utilizado foi a versão curta do Questionário Internacional de Atividade Física (IPAQ), em 50,00\% dos estudos ( $\mathrm{n}=4$ ). Nos estudos realizados no SUS, 37,5\% $(n=3)$ utilizaram a versão curta do IPAQ e quando questionado sobre o modo de aconselhamento para AF nas UBS não ocorreu um consenso entre os estudos (Dados não mostrados).

Em relação às avaliações dos programas de $\mathrm{AF}$, os artigos selecionados indicaram que ao menos uma avaliação foi realizada, seja esta por meio de dados descri$\operatorname{tivos}^{11,12-14,19,20}$, do processo ${ }^{21,22}$, do impacto ${ }^{21-25}$ e/ou resultados ${ }^{21,23,24-31}$. 


\section{Programa Academia da Cidade}

O Programa Academia da Cidade (PAC) teve início no ano de 2002. É um programa gratuito de promoção da saúde vinculado à secretaria de saúde de Recife que ocorrem junto ao Sistema Único de Saúde. As atividades são desenvolvidas durante a semana (segunda a sexta feira) em dois turnos, pela manhä (05 horas às 10 horas) e no período noturno (17:00 as 22:00 horas) em praças, parques, avenidas e na praia ${ }^{22,25}$

\section{Serviço de Orientação e Exercício}

O programa Serviço de Orientação e Exercício (SOE) é realizado desde 1990, sendo o principal objetivo elevar o níveis de atividade física principalmente da populaçẫo mais necessitada. É realizado em parques, praças ou em qualquer lugar que possa promover atividade física, sempre acompanhada por um profissional de educação física. 0 programa combina estratégias de mudanças de comportamento a açōes educativa ${ }^{24}$.

\section{CuritibAtiva}

0 programa CuritibAtiva teve seu início em 1998 devido ao aumento no número de pessoas com DCNT's tendo como objetivo elevar os níveis de atividade física e melhorar a qualidade de vida da população. 0 programa é desenvolvido pela secretária de esporte lazer e pela secretária de saúde do município. As atividades säo desenvolvidas em espaços públicos da cidade (departamentos de esportes, parques, praças, academia, piscinas e ciclovias eciclofaixas) $23,2.2$

\section{Saúde Ativa Rio Claro}

- o programa Saúde Ativa Rio Claro teve início em 2001 sendo desenvolvido pelo núcleo de Atividade Física, Esporte e Saúde da Universidade de Rio Claro-SP em parceira com departamento municipal de saúde. O programa é desenvolvido para mulheres, com idade superior a 50 anos com baixonivel socioeconômico. 0 principal objetivo do programa é elevar os niveis de atividade física, proporcionar melhorias na percepção de saúde e qualidade de vida da população

\section{Projeto Viver Saudáve}

O Projeto Viver Saudável foi implementado em 2007 pela secretária municipal de saúde com objetivo de estimular condutas que viabilizem estilo de vida saudável, tais como prática regular de atividade física, escolhas alimentares adequadas e abandono de práticas nocivas à saúde, como tabagismo, consumo de álcool em escolares de rede pública de ensino ${ }^{33}$.

\section{Ação Saúde Floripa educar, concientizar e praticar}

Programa "Ação e Saúde Floripa-educar, conscientizar e praticar" ocorreu durante 120 dias no ano de 2006. Foi desenvolvido em unidades de saúde, adotando a estratégia e açōes multiprofissionais. Foi entregue para a população quartilhas contendo orientação sobre a prática da atividade física ${ }^{29}$.

FIGURA 4 - Descrição dos programas inseridos na revisão sistemática.

Os estudos que apresentaram apenas informações descritivas, relataram aumento no número de programas de AF oferecidos para a população nos anos de 2005 à 2009 e no aconselhamento da AF entre médicos e enfermeiros nas Unidades Básicas de Saúde (UBS) ${ }^{11,12-14,19,20}$.

Dois estudos avaliaram o processo de implementação de programas. Na cidade de Curitiba, no programa CuritibAtiva, a maioria dos entrevistados indicaram como ótima/boa a qualidade das estruturas oferecidas e apenas 10,00\% receberam material de divulgação (folder) sobre o programa ${ }^{22}$. Em Pernambuco, no Programa Academia da Cidade, os frequentadores $(72,80 \%)$ reportaram estar satisfeitos com o programa, porém $61,00 \%$ relataram que gostariam de uma melhor estrutura e aquisição de novos materiais, $8,70 \%$ gostariam da ampliação do serviços e $2,30 \%$ contratação de profissionais e maior divulgação ${ }^{21}$.

A avaliação de impacto foi verificada através do conhecimento dos programas oferecidos. O programa Serviço de Orientação ao Exercício (SOE), realizado na cidade de Vitória-ES, sendo este o estudo que apresentou o menor percentual de conhecimento $(31,50 \%)$ por parte dos entrevistados ${ }^{23}$. Em contrapartida, na cidade 
de Curitiba-PR, o Programa CuritibAtiva foi o que apresentou o maior percentual $(91,60 \%)^{25}$. Em relação ao delineamento dos estudos, todos foram transversais.

Para avaliação dos resultados, a principal variável investigada foi o aumento nos níveis de Atividade Física no Lazer (AFL). Os frequentadores que participavam dos programas eram mais ativos fisicamente no tempo livre e em caminhadas, quando comparados com aqueles que não participavam ou não conheciam os programas ${ }^{21,23,25,26}$. Do mesmo modo, no programa Academia da Cidade, Simões et al. ${ }^{24}$ encontraram que os ex-participantes eram duas vezes mais propensos a praticarem Atividade Física Moderada e Vigorosa no Lazer (AFMVL) quando comparado com aqueles que nunca participaram do programa.

Nos estudos realizados em UBS, o recebimento de aconselhamento para AF foi mais reportado entre aqueles que possuem hipertensão arterial sistêmica e excesso de peso, utilizam medicamentos e frequentam o programa Academia da Saúde ${ }^{31}$. Nos estudos desenvolvidos na cidade de Rio Claro-SP, os frequentadores do programa reportaram melhorias na percepção de qualidade de vida, redução do número de visitas nas UBS e a redução na pressão arterial e glicemia ${ }^{29}$. Para os aspectos da aptidão física relacionados à saúde, a coordenação motora, agilidade e força muscular apresentaram melhoras significativas para os participantes com idade $\geq 70$ anos, exceto a capacidade aeróbica e flexibilidade ${ }^{27}$.

\section{Discussão}

O objetivo deste estudo foi sintetizar as evidências disponíveis na literatura referentes aos programas para promoção de AF no Sistema Único de Saúde brasileiro. Através da estratégia de busca, foram selecionados 17 estudos. Os meios de promoção mais realizados foram ações de promoção da AF e prevenção de DCNTs junto ao SUS (47,05\%; $\mathrm{n}=8)$ e programas com equipe multiprofissional $(47,05 \%$; $\mathrm{n}=8)$. A maior parte dos estudos $(74,47 \%)$ incluiu pessoas com idade $\geq 18$ anos. Observou-se ainda disparidade regional de publicações, sendo a maior proporção de estudos na região sudeste. Finalmente, os estudos foram em sua maioria descritivos ou sobre resultados na AF ou qualidade de vida, sendo escassas as informações sobre processo e impacto dos programas.

A promoção da AF é uma das prioridades de saúde pública em todo o mundo, principalmente, em países de média e baixa rendas ${ }^{32}$. Nós últimos anos, observouse um avanço na produção científica na área de promoção de $\mathrm{AF}$ no Brasil ${ }^{33}$ e esse crescimento se reflete no crescimento em quantidade dos estudos de promoção de AF no SUS conforme os achados deste estudo.

O presente estudo identificou uma concentração de publicações na região sudeste. Talvez, isso possa ser explicado pelo maior número de pesquisadores, centros de pesquisa e cursos de pós-graduação nessa região ${ }^{34}$. Apesar do incentivo do Governo Federal para promoção da AF no SUS ${ }^{9,14}$, a nível nacional, não foram encontradas publicações do principal programa de promoção de AF no Brasil "Academia da Saúde" 10,35 , idealizado no ano de 2011 com previsão de construção de 4.000 polos até o ano $2015^{35}$. Neste sentido, é importante ressaltar a necessidade que os pesquisadores desenvolvam estudos que descrevam o perfil dos frequentadores, modo de intervenção e avaliem o custo-efetividade deste programa, em todas as regiões do país.

Outro programa desenvolvido no SUS, é o aconselhamento da AF por uma equipe multiprofissional nas UBS junto ao Núcleo de Apoio da Saúde da Família $(\mathrm{NASF})^{19}$. Os achados desta revisão mostraram que o aconselhamento está entre os principais meios de promoção da AF no SUS, corroborando com a revisão de Costa et $\mathrm{al}^{36}$, onde mostra que os agentes comunitários de saúde tem um papel 
importante na promoção da AF, seja ela feita através de aconselhamentos, reuniões de grupo, intervenções específicas, reeducação alimentar, flipcharts, revistas e vídeos ${ }^{6,36}$. Contudo, os profissionais de saúde, reportaram várias barreiras para realizá-la, como falta de tempo, conhecimento e de protocolos que descrevam a melhor maneirar de promover $\mathrm{AF}^{37}$. Talvez, a inserção do profissional de educação física no SUS possa ser um facilitador para a promoção da $\mathrm{AF}^{44}$, desde que estes conteúdos sejam abordados durante o processo de formação destes profissionais.

As principais atividades físicas desenvolvidas para a promoção de AF no SUS encontradas no presente estudo foram caminhadas em grupo e atividades físicas desenvolvidas em espaços públicos. Programas que envolveram uma grande quantidade de pessoas, como programas comunitários, foram classificados como ações promissoras para promoção da $\mathrm{AF}^{45,46}$. Duas revisões recentes identificaram que a criação de locais para a prática de AF e aulas em grupos foi uma excelente maneira de elevar os níveis de $\mathrm{AF}^{47,48}$. Em países de alta renda, como Canadá e Estados Unidos, o desenvolvimento de programas em parques e praças tem elevado o nível de AF da população ${ }^{47}$. Resultados semelhantes também foram encontrados em países da América Latina (Colômbia ${ }^{47}$ e Brasi1 $^{28,49}$ ). Contudo, pouco se sabe sobre a efetividade dos programas e quais desses podem ser adaptados em outros contex$\operatorname{tos}^{48}$. Nesse sentido, sugere-se melhorias na avaliação dos programas de AF (processo de implementação, impacto e resultados) ${ }^{19,48}$. Por exemplo: todos estudos encontrados nessa revisão, foram estudos transversais, o que limita a avaliação de resultado e de impacto dos programas. Desse modo, recomenda-se realizar estudos com delineamentos longitudinais ou mesmo experimentais, acerca do custo e benefício dos programas implementados.

Os dados da presente revisão indicam que os programas de promoção da AF foram realizados principalmente para a população com idade $\geq 18$ anos. Talvez, isso ocorra pelo fato de dois terços da população adulta não cumprirem as recomendações de AF e o elevado número de mortes relacionadas as DCNTs no Brasil ${ }^{50}$. Além disso, o fato da população adulta buscar de maneira mais frequente as UBS, pode parcialmente explicar o enfoque dos programas para este grupo populacional. Contudo, programas desenvolvidos para crianças e adolescentes, no contexto escolar, como o programa Saúde na Escola, também são estratégias importantes para promoção de saúde e o aumento dos níveis de $\mathrm{AF}^{51}$ destes grupos etários. Evidências suportam que os programas realizados na escola são os únicos baseados em evidências no Brasil ${ }^{45,52}$, e que a realização destes, pode contribuir para uma vida ativa fisicamente na idade adulta. Entretanto, apenas um estudo incluído nesta revisão foi realizado no âmbito escolar. Dessa forma, recomenda-se que futuros estudos, busquem avaliar as implicações para a promoção da AF do Programa Saúde na Escola.

Em relação ao modo de mensuração de AF, o instrumento mais utilizado foi a versão curta do Questionário Internacional de Atividade Física (IPAQ). Este instrumento foi adaptado e validado para o contexto brasileiro, é de fácil aplicação e de baixo custo ${ }^{53,54}$. Todavia, recomenda-se o uso da versão longa do instrumento. Pois este, permite compreender de modo específico os domínios da AF (lazer, deslocamento, trabalho e ocupação) na população. Desse modo, permitirá identificar em qual domínio a população possui maior ou menor nível de AF e assim auxiliar no planejamento, desenvolvimento e implementação de programas. Além disto, o IPAQ curto pode superestimar os níveis de AF o que pode limitar as repercussões dos programas para a saúde da população. Outro ponto a ser destacado, é que futuros estudos adotem medidas padronizadas para avaliação da AF, além de utilizar medidas mais precisas como o uso de acelerômetros. 
Algumas limitações devem ser consideradas para a melhor compreensão dos resultados. a) a busca limitou-se a estudos originais e indexados nas bases de dados, excluindo principalmente relatórios, que podem ser fontes importantes para fornecer resultados sobre os programas de AF realizados no SUS, porém para manter o rigor metodológico e qualidade dos conteúdos selecionados optou-se por incluir apenas estudos originais; b) diferentes delineamentos e metodologias aplicadas nos estudos impossibilitou a comparação entre os método de promoção de AF realizados no SUS, talvez estudos específicos com intervenções ou aplicação de uma metodologia e programas semelhantes, em diferentes localidades, permitirá a comparação dos resultados; c) em alguns estudos o impacto do programa foi avaliado através da comparação de participantes expostos e não expostos ao programa, produzindo um viés nos achados, limitando a avaliação do impacto para população como um todo; d) a heterogeneidade dos estudos, impossibilitou selecionar uma ferramenta que avaliasse a qualidade metodológica dos estudos inseridos na revisão sistemática.

Esta revisão sistemática foi a primeira a sintetizar evidências sobre os programas de promoção da AF do SUS no Brasil. Tais achados permitem concluir que programas comunitários e aconselhamentos são as principais ações para a promoção da $\mathrm{AF}$ no SUS, principalmente na população adulta ( $\geq 18$ anos). Além disto, as publicações têm sido conduzidas, em sua grande maioria, na região sudeste do país. Os estudos, em sua maioria forneceram dados descritivos ou sobre resultados na AF ou qualidade de vida, sendo escassas as informações sobre processo e impacto dos programas. Recomenda-se que futuros estudos aprimorem o modo de avaliação (processo, impacto e resultados). Além disso, sugere-se realizar estudos com delineamentos longitudinais ou mesmo experimentais, acerca do custo e benefício dos programas implementados e posteriormente, políticas públicas, que incentivem a promoção da AF, poderão ser desenvolvidas bem como a expansão dos programas já existentes.

\section{Contribuição dos autores}

Leonardo Augusto Becker, participou na fase de concepção, revisão da literatura, redação e versão final do artigo. Priscila Bezerra Gonçalves, participou na concepção, redação e versão final do artigo. Rodrigo Siqueira Reis, contribuiu na concepção e revisão crítica do artigo.

\section{Referências}

1. Hallal PC, Andersen LB, Bull FC, Guthold R, Haskell W, Ekelund U. Global physical activity levels: surveillance progress, pitfalls, and prospects. Lancet. 2012; 380: 247-57.

2. Lee I-M, Shiroma EJ, Lobelo F, Puska P, Blair SN, Katzmarzyk PT. Effect of physical inactivity on major non-communicable diseases worldwide: an analysis of burden of disease and life expectancy. Lancet. 2012; 380: 219-29.

3. Kohl HW, Craig CL, Lambert EV, Inoue S, Alkandari JR, Leetongin G, et al. The pandemic of physical inactivity: global action for public health. Lancet. 2012; 380: 294-305.

4. Pratt M, Norris J, Lobelo F, Roux L, Wang G. The cost of physical inactivity: moving into the 21st century. Br J Sports Med. 2014; 48: 171-3.

5. Sanchez A, Bully P, Martinez C, Grandes G. Effectiveness of physical activity promotion interventions in primary care: a review of reviews. Elsevier. 2015 DOI:10.1016/j. ypmed.2014.09.012.

6. Orrow G, Kinmonth A-L, Sanderson S, Sutton S. Effectiveness of physical activity promotion based in primary care: systematic review and meta-analysis of randomised controlled trials. BMJ. 2012; 344: e1389.

7. Taggart J, Williams A, Dennis S, Newall A, Shortus T, Zwar N, et al. A systematic review of interventions in primary care to improve health literacy for chronic disease behavioral risk factors. BMC Fam. Pract. 2012; 13: 49.

8. Vuori IM, Lavie CJ, Blair SN. Physical activity promotion in the health care system. Mayo Clin Proc. 2013; 88: 1446-61. 
9. Malta D, Silva M, Albuquerque G, Amorim R, Rodrigues G, Silva T, et al. Política Nacional de Promoção da Saúde, descrição da implementação do eixo atividade física e práticas corporais, 2006 a 2014. Rev Bras Ativ Fís Saúde. 2014; 19: 286-99.

10. Malta DC, Silva Jr JB Da. O Plano de Ações Estratégicas para o Enfrentamento das Doenças Crônicas Não Transmissíveis no Brasil e a definição das metas globais para o enfrentamento dessas doenças até 2025: uma revisão. Epidemiol Serv Saúde. 2013; 22: 151-64.

11. Ramos LR, Malta DC, Gomes GA de O, Bracco MM, Florindo AA, Mielke GI, et al. Prevalence of health promotion programs in primary health care units in Brazil. Rev Saude Publica. 2014; 48: 837-44.

12. Ramos LR, Malta DC, Gomes GA de O, Bracco MM, Florindo AA, Mielke GI, et al. Prevalence of health promotion programs in primary health care units in Brazil. Rev Saude Publica. 2014; 48: 837-44.

13. Florindo AA, Mielke GI, Gomes GADO, Ramos LR, Bracco MM, Parra DC, et al. Physical activity counseling in primary health care in Brazil: a national study on prevalence and associated factors. BMC Public Health. 2013; 13: 794.

14. Knuth AG, Malta DC, Cruz DK, Castro AM, Fagundes J, Sardinha LM, et al. Description of the countrywide physical activity network coordinated by the Brazilian Ministry of Health: 2005-2008. J Phys Act Health. 2010; 7 Suppl 2 : S253-8.

15. Amorin TC, Knuth AG, Cruz DKA, Reis RS, Hallal PC. Descrição dos programas municipais de promoção da atividade física financiados pelo Ministério da Saúde Description of the physical activity. Rev Bras Ativ Fís Saúde. 2013; 18: 63-74.

16. Moher D, Liberati A, Tetzlaff J, Altman DG. Preferred Reporting Items for Systematic Reviews and Meta-Analyses : Ann Intern Med. 2014; 151: 264-9.

17. Malta DC, Castro AM De, Gosch CS, Cruz DKA, Bressan A, Nogueira JD, et al. A Política Nacional de Promoção da Saúde e a agenda da atividade física no contexto do SUS National. Epidemiol Serv Saúde. 2009; 18: 79-86.

18. Global Advocacy for Physical Activity (GAPA) the Advocacy Council of the International Society fpt Physical Activity and Health (ISPAH). Non Communicable Disease Prevention and Control (NPC): Investimens that work for Physical Activity. 2011.

19. Brownson RC, Baker EA, Leet TL, GillesPie KN, True WR. Evidence-Based Public Health, Publisher: Oxford University Press. Second Edi. 2011.

20. Gomes GA de O, Kokubun E, Mieke GI, Ramos LR, Pratt M, Parra DC, et al. Characteristics of physical activity programs in the Brazilian primary health care system. Cad Saude Publica. 2014; 30: 2155-68.

21. Siqueira FV, Nahas MV, Facchini LA, Silveira DS, Piccini RS, Tomasi E, et al. Aconselhamento para a prática de atividade física como estratégia de educação à saúde. Cad Saude Publica. 2009; 25: 203-13.

22. Hallal PC, Tenorio MCM, Tassitano RM, Reis RS, Carvalho YM, Cruz DKA, et al. Evaluation of the Academia da Cidade program to promote physical activity in Recife, Pernambuco State, Brazil: perceptions of users and non-users. Cad Saude Publica. 2010; 26: 70-8.

23. Hallal P, Reis R, Hino A. Avaliação de programas comunitários de promoção da atividade física: o caso de Curitiba, Paraná; Evaluation of community physical activity programs: the case of. Rev Bras Ativ Fís Saúde. 2009; 14: 104-14.

24. Reis RS, Hino AAF, Cruz DK, da Silva Filho LE, Malta DC, Domingues MR, et al. Promoting physical activity and quality of life in Vitoria, Brazil: evaluation of the Exercise Orientation Service (EOS) program. J Phys Act Health. 2014; 11: 38-44.

25. Simoes EJ, Hallal P, Pratt M, Ramos L, Munk M, Damascena W, et al. Effects of a community-based, professionally supervised intervention on physical activity levels among residents of Recife, Brazil. Am J Public Health. 2009; 99 : 68-75.

26. Reis RS, Hallal PC, Parra DC, Ribeiro IC, Brownson RC, Pratt M, et al. Promoting physical activity through community-wide policies and planning: findings from Curitiba, Brazil. J Phys Act Health. 2010; 7 (Suppl 2) : S137-45.

27. Hallal PC, Tenório MCM, Tassitano RM, Reis RS, Carvalho YM, Cruz DKA, et al. Avaliação do programa de promoção da atividade física Academia da Cidade de Recife, Pernambuco, Brasil: percepções de usuários e não-usuários. Cad Saude Publica. 2010; 26: 70-8.

28. Reis RS, Hallal PC, Parra DC, Ribeiro IC, Brownson RC, Pratt M, et al. Promoting Physical Activity Through Community-Wide Policies and Planning: Findings From Curitiba , Brazil. J Phys Act Health. 2010; 7: 137-45.

29. Gomes MDEA, Fátima MDE, Silva DA. Efetividade de uma intervenção de atividade física em adultos atendidos pela estratégia saúde da família: programa ação e saúde floripa - Brasil. Rev Bras Ativ Fís. Saúde. 2008; 13:1.

30. Nakamura PM, Papini CB, Teixeira IP, Chiyoda A, Luciano E, Cordeira KL, et al. Effect on Physical Fitness of a 10-Year Physical Activity Intervention in Primary Health Care Settings. J Phys Act Health. 2015; 12: 102-8. 
31. Papini CB, Nakamura PM, Zorzetto LP, Thompson JL, Phillips AC, Kokubun E. The effect of a community-based, primary health care exercise program on inflammatory biomarkers and hormone levels. Mediators Inflamm. 2014; 2014: 185-707.

32. Giraldo A, Gomes G, Serafim T, Zorzeto L, Aquino D, Kokubun E. Influence of a physical activity program on the use of Primary Care services in the city of Rio Claro, SP. Rev Bras Ativ Fís. Saúde. 2013; 18: 186-96.

33. Ferreira JO, Jardim PCBV, Peixoto MDRG. Avaliacao de projeto de promoção da saúde para adolescentes. Rev Saude Publica. 2013; 47: 257-65.

34. Lopes ACS, Toledo MTT De, Câmara AMCS, Menzel H-JK, Santos LC Dos. Condições de saúde e aconselhamento sobre alimentação e atividade física na Atenção Primária à Saúde de Belo Horizonte-MG. Epidemiol Serv Saúde. 2014; 23: 305-16.

35. Hallal PC, Tenorio MCM, Tassitano RM, Reis RS, Carvalho YM, Cruz DKA, et al. Evaluation of the Academia da Cidade program to promote physical activity in Recife, Pernambuco State, Brazil: perceptions of users and non-users. Cad Saude Publica. 2010; 26: 70-8.

36. Nakamura PM, Papini CB, Teixeira IP, et al. Effect on physical fitness of a 10-year physical activity intervention in primary health care settings. J Phys Act Health. 2015; 12: 102-8.

37. WHO. Global Status Report On Noncommunicable Diseases 2014. In: World Health Organization. 2014: 1-280.

38. Ramires V, Becker L, Sadovsky A, Zago A, Bielemann R, Guerra P. Evolução da pesquisa epidemiológica em atividade física e comportamento sedentário no Brasil: atualização de uma revisão sistemática. Rev Bras Ativ Fís Saúde. 2014; 19: 453-60.

39. Silva B, Rey-López J, Hartwig F, Barbosa-Silva T, Bielemann R, Coll C. A contribuição do Brasil para a pesquisa em atividade física e saúde no cenário internacional. Rev Bras Ativ Fís Saúde. 2014; 19. DOI:10.12820/rbafs.v.19n5p618.

40. Brasil. Ministério da Saúde. Portaria no 719, de 07 de Abril de 2011. Cria o programa academia da saúde no âmbito do sistema único de saúde. 2011.

41. Gomes GA de O, Kokubun E, Mieke GI, et al. Characteristics of physical activity programs in the Brazilian primary health care system. Cad Saude Publica. 2014; 30: 2155-68.

42. Costa EF, Guerra PH, Santos TI Dos, Florindo AA. Systematic review of physical activity promotion by community health workers. Prev Med. 2015; 81: 114-21.

43. Hébert ET, Caughy MO, Shuval K. Primary care providers' perceptions of physical activity counselling in a clinical setting: a systematic review. Br J Sports Med. 2012; 46: 625-31.

44. Souza SC, Loch MR. Intervenção do profissional de educação física nos Núcleos de Apoio à Saúde da Família em municípios do norte do Paraná. Rev Bras Ativ Fís Saúde. 2011; 16: 5-10.

45. Hoehner CM, Ribeiro IC, Parra DC, et al. Physical activity interventions in Latin America: expanding and classifying the evidence. Am J Prev Med. 2013; 44: e31-40.

46. Heath GW, Parra DC, Sarmiento OL, et al. Evidence-based intervention in physical activity: lessons from around the world. Lancet.; 380: 272-81.

47. Pratt M, Perez LG, Goenka S, et al. Can Population Levels of Physical Activity Be Increased? Global Evidence and Experience. Prog Cardiovasc Dis. 2015; 57: 356-67.

48. Heath GW, Parra DC, Sarmiento OL, et al. Evidence-based intervention in physical activity: Lessons from around the world. Lancet. 2012; 380: 272-81.

49. Reis RS, Hino AAF, Cruz DK, et al. Promoting physical activity and quality of life in Vitoria, Brazil: evaluation of the Exercise Orientation Service (EOS) program. J Phys Act Health. 2014; 11: 38-44.

50. VIGITEL. Ministério da Saúde. Secretária de Vigilãncia em Saúde. VIGITEL Brasil 2013: Vigilânica de Fatores de riscos e proteção para doenças crônicas por inquérito telefônico. Brasil: Ministério da Saúde 2014.

51. Brasil. Ministério da Saúde. Decreto no 6.286, de 5 de dezembro de 2007. Institui no âmbito dos Ministérios da Educação e da Saúde, o Programa Saúde na Escola. Ministério da Educ. e da Saúde. 2007.

52. Hoehner CM, Soares J, Perez DP, et al. Intervenções em Atividade Física na América Latina Uma revisão Sistemática. Am J Prev Med. 2008; 34: 224-33.

53. Craig CL, Marshall AL, et al. International Physical Activity Questionnaire: 12-Country Reliability and Validity. Med Sci Sport Exerc. 2003; 35: 1381-95.

54. Hallal PC, Gómez LF, Parra DC, et al. Lecciones aprendidas después de 10 Años del uso de IPAQ en Brasil y Colombia. J Phys Act Health. 2010; 7: 259-64.

ENDEREÇO PARA

CORRESPONDÊNCIA LEONARDO AUGUSTO BECKER leonardobecker26@hotmail.com
Pontifícia Universidade Católica do

Paraná. Escola de Saúde e Biociências.

Grupo de Pesquisa em Atividade Física

e Qualidade de Vida (GPAQ)

Tel: + 554132712503

Rua Imaculada Conceição, 1155 - Prado

Velho-80215-901-Curitiba-PR, Brasil. 